\title{
Effect of xenon and dexmedetomidine as adjuncts for general anesthesia on postoperative emergence delirium after elective cardiac catheterization in children: study protocol for a randomized, controlled, pilot trial
}

Sarah Devroe ${ }^{1,2^{*}} \mathbb{D}$, Lisa Devriese ${ }^{1}$, Frederik Debuck' ${ }^{1}$ Steffen Fieuws ${ }^{3}$, Bjorn Cools ${ }^{2,4}$, Marc Gewillig ${ }^{2,4}$, Marc Van de Velde ${ }^{1,4}$ and Steffen Rex ${ }^{1,4}$

\begin{abstract}
Background: Emergence delirium, a manifestation of acute postoperative brain dysfunction, is frequently observed after pediatric anesthesia and has been associated with the use of sevoflurane. Both xenon and dexmedetomidine possess numerous desirable properties for the anesthesia of children with congenital heart disease, including hemodynamic stability, lack of neurotoxicity, and a reduced incidence of emergence delirium. Combining both drugs has never been studied as a balanced-anesthesia technique. This combination allows the provision of anesthesia without administering anesthetic drugs against which the Food and Drug Administration (FDA) issued a warning for the use in young children.

Methods/Design: In this phase-II, mono-center, prospective, single-blinded, randomized, controlled pilot trial, we will include a total of 80 children aged 0-3 years suffering from congenital heart disease and undergoing general anesthesia for elective diagnostic and/or interventional cardiac catheterization. Patients are randomized into two study groups, receiving either a combination of xenon and dexmedetomidine or mono-anesthesia with sevoflurane for the maintenance of anesthesia.

The purpose of this study is to estimate the effect size for xenon-dexmedetomidine versus sevoflurane anesthesia with respect to the incidence of emergence delirium in children. We will also describe group differences for a variety of secondary outcome parameters including peri-interventional hemodynamics, emergence characteristics, incidence of postoperative vomiting, and the feasibility of a combined xenon-dexmedetomidine anesthesia in (Continued on next page)
\end{abstract}

\footnotetext{
* Correspondence: sarah.devroe@uzleuven.be

'Department of Anesthesiology, University Hospitals Leuven, Herestraat 49, 3000 Leuven, Belgium

${ }^{2}$ Department of Pediatric and Congenital Cardiology, University Hospitals Leuven, Leuven, Belgium

Full list of author information is available at the end of the article
}

(c) The Author(s). 2020 Open Access This article is licensed under a Creative Commons Attribution 4.0 International License, which permits use, sharing, adaptation, distribution and reproduction in any medium or format, as long as you give appropriate credit to the original author(s) and the source, provide a link to the Creative Commons licence, and indicate if changes were made. The images or other third party material in this article are included in the article's Creative Commons licence, unless indicated otherwise in a credit line to the material. If material is not included in the article's Creative Commons licence and your intended use is not permitted by statutory regulation or exceeds the permitted use, you will need to obtain permission directly from the copyright holder. To view a copy of this licence, visit http://creativecommons.org/licenses/by/4.0/ The Creative Commons Public Domain Dedication waiver (http://creativecommons.org/publicdomain/zero/1.0/) applies to the data made available in this article, unless otherwise stated in a credit line to the data. 


\section{(Continued from previous page)}

children.

Discussion: Sevoflurane is the most frequently used anesthetic in young children, but has been indicated as an independent risk factor in the development of emergence delirium. Xenon and dexmedetomidine have both been associated with a reduction in the incidence of emergence delirium. Combining xenon and dexmedetomidine has never been described as a balanced-anesthesia technique in children. Our pilot study will therefore deliver important data required for future prospective clinical trials.

Trial registration: EudraCT, 2018-002258-56. Registered on 20 August 2018. https://www.clinicaltrialsregister.eu.

Keywords: Anesthetics, Inhalation, Xenon, Sevoflurane, Dexmedetomidine, Emergence delirium, Pediatric anesthesia

\section{Background}

Emergence delirium (ED) is a manifestation of acute postoperative brain dysfunction that occurs with a relatively high frequency after pediatric anesthesia. The incidence varies depending on the diagnostic criteria used and the combination of administered anesthetic drugs [1]. It can be associated with either short-term complications, such as an increased risk of injury in children, parental dissatisfaction, increased costs, and length of hospital stay, or potential long-term consequences, including persistent psychological and behavior problems [2]. While the exact pathophysiological mechanisms underlying ED remain unknown, the use of sevoflurane has been identified as one of the most important risk factors [3, 4] There is a continuous search for anesthetics that ameliorate or avoid the occurrence of emergence agitation [2].

In children with congenital heart disease (CHD), therapeutic decisions are often based on the hemodynamic parameters measured during cardiac catheterization. The ideal anesthetic technique for this procedure should maintain hemodynamics that reflect awake baseline conditions to provide the best therapeutic option. Unfortunately, most anesthetics affect myocardial function and the vascular tone, and the ideal anesthetic regimen for these procedures remains a subject of debate.

In adults, xenon anesthesia preserves baseline blood pressure (BP) better than volatile anesthetics or propofol [5]. Moreover, xenon has been repeatedly demonstrated to offer neuroprotection in various animal models and models of neuronal injury [6]. More specifically, xenon has been demonstrated to attenuate isoflurane-induced neurotoxicity in rats [7]. Whereas xenon can be used as a mono-anesthetic in adults, it can possibly only be used as an additive to other anesthetics in children. The minimum alveolar concentration (MAC) of xenon in children is unknown but, concurring with other anesthetics, is expected to be higher than in adults (MAC 63.1\%) [8]. In a meta-analysis, the MAC of xenon for children at the age of 1 year was calculated to be $92 \%$ [9]. As a consequence, xenon can only be used in sub-anesthetic concentrations for children to guarantee an inspiratory oxygen concentration of at least $30 \%$. While subanesthetic concentrations of xenon were demonstrated to be neuroprotective [10], they need to be supplemented by another anesthetic or sedative drug to achieve sufficient depth of anesthesia.

We previously demonstrated in children with CHD that the addition of $60 \%$ xenon to sevoflurane resulted in a $60 \%$ reduction of the mean expiratory sevoflurane concentration of sevoflurane to achieve a comparable depth of anesthesia $[11,12]$. Moreover, we did find a decreased incidence of ED when xenon was added to sevoflurane anesthesia [11]. However, we could not demonstrate increased hemodynamic stability. Most probably, the hemodynamic advantages known from adults receiving mono-xenon anesthesia were masked by the necessary addition of sevoflurane to achieve an acceptable depth of anesthesia in children receiving xenon.

The ideal drug to add to xenon for balanced anesthesia should potentiate the anesthetic effects of xenon without jeopardizing hemodynamic stability.

Due to its sedative, anxiolytic, sympatholytic, and analgesic properties, dexmedetomidine has recently been introduced in different areas of pediatric anesthesia as an adjunct for balanced anesthesia, for premedication or as part of sedation techniques. Moreover, a recent systematic review and meta-analysis in pediatric patients undergoing congenital cardiac surgery found the administration of dexmedetomidine to result in more stable intraoperative hemodynamics, a lower incidence of ED, and an attenuated stress response [13-15]. A sparing effect of the MAC for sevoflurane has been demonstrated when an initial bolus of dexmedetomidine was followed by an infusion [16]. Moreover, the infusion of dexmedetomidine produced a dose-dependent decrease in the amount of sevoflurane required to produce $50 \%$ excellent tracheal intubation conditions in children [17]. Notably, like xenon, dexmedetomidine was also found to have neuroprotective properties in preclinical research. More specifically, it was observed that dexmedetomidine protects against anesthesia-induced neurotoxicity [18]. 
Altogether we can conclude that both xenon and dexmedetomidine possess numerous desirable properties with respect to the treatment of pediatric patients with $\mathrm{CHD}$, including a benign hemodynamic profile $[5,14,15]$, a reduced catecholamine release, anesthetic-sparing effects, a decreased incidence of ED, and neuroprotective effects [13]. This innovative combination of xenon and dexmedetomidine could be of particular interest for pediatric anesthesia, as combining these anesthetics has never been described as a balanced-anesthesia technique in children. Moreover, pediatric data on both drugs are scarce and, therefore, the FDA had no evidence to include these products in the "list of general anaesthetic and sedation drugs" for which a label change was requested indicating that "the repeated or lengthy use during surgeries or procedures in children younger than three years or in pregnant women during their third trimester may affect the development of children's brains" [19].

\section{Methods/Design}

Aim of the study

The primary aim of this study is to describe the effect size for xenon-dexmedetomidine versus sevoflurane anesthesia with respect to the incidence of emergence delirium in children with $\mathrm{CHD}$ undergoing elective diagnostic/interventional cardiac catheterization. The secondary aim is to estimate group differences for a variety of secondary outcome parameters including periinterventional hemodynamics, emergence characteristics, incidence of postoperative vomiting, and the feasibility of a combined xenon-dexmedetomidine anesthesia in children.

\section{Design of the study}

The design of this study is reported in accordance with the SPIRIT 2013 Checklist: Recommended items to address in a clinical trial protocol (Additional file 1) [20].

This prospective, randomized, controlled, observerblinded trial is performed at the University Hospitals of the KU Leuven by two types of investigators. Investigator I assesses the primary outcome (and the majority of the secondary outcome parameters) and is blinded to the group affiliation. Investigator II performs anesthesia and cannot be blinded to the treatment groups due to the mandatory monitoring of anesthetic concentrations. As a consequence, this study has to be performed observer-blinded.

The trial is conducted in compliance with the principles of the Declaration of Helsinki, the Principles of Good Clinical Practice, and in accordance with all applicable regulatory requirements. The protocol (version SD-DXP: 2 06-08-2018) and related documents were approved by the Ethics Committee of the University
Hospitals Leuven (S61690 August 18th 2018) and the Federal Agency for Medicines and Health Products, Brussels, Belgium for Clinical Trial Authorization (FAGG/R\&D/BEN/ben 1,111,021; 20 August 2018). The study is registered in the European Clinical Trails Database of the European Medicines Agency (EudraCT: 2018-002258-56) (see Additional file 2 for additional trial registration items).

To avoid selection bias, randomization and allocation concealment will be used. Patients are randomized to one of the study groups using a software-generated allocation sequence (RandList, V. 1.2). Group assignment is ensured by using sealed, opaque, sequentially numbered envelopes, which are opened only after arrival of the patient in the interventional room. As mentioned above, the attending anesthesiologist cannot be blinded for the treatment allocation. Consequently, a code break is not necessary.

\section{Inclusion and exclusion criteria}

We screen all consecutive children scheduled for elective (diagnostic or therapeutic) heart catheterization under general anesthesia. We include children aged 1 month to 3 years.

Exclusion criteria comprise the lack of parental informed consent, a cyanotic congenital heart defect possibly requiring a $\mathrm{FiO}_{2}$ of $>50 \%$ during the procedure, high-risk and complex interventional procedures (as defined by the pediatric cardiologist), evidence of behavioral or cognitive impairment, and the presence of a contraindication for the use of one of the investigated drugs.

\section{Outcome parameters \\ Primary endpoint}

- Incidence of ED as assessed by the Watcha-scale (4point agitation scale) (Table 1) [21, 22]. A patient will be classified as having ED in case of a Watcha-scale $\geq$ 3. Moreover, every patient will also be tested using the "Pediatric Anesthesia Emergence Delirium Scale" (PAED scale) (Table 2). A patient will be classified as having ED in case of a PAED scale $\geq 10[1,15,17]$. In case of conflicting observations between the Watcha and the PAED scale, the results of the Watcha scale will be the crucial factor for the diagnosis of ED.

Table 1 WATCHA scale or 4-point agitation score [21]

WATCHA Scale

Child is calm

Child is crying but can be consoled

Child is crying and cannot be consoled Child is agitated and thrashing around 
Table 2 PAED score [23]

\begin{tabular}{|c|c|c|c|c|c|c|}
\hline \multicolumn{7}{|c|}{ PAED-scoring } \\
\hline Point & Description of item & Not at all & Just a little & Quite a bit & Very much & Extremely \\
\hline 1 & Child makes eye contact with caregiver & 4 & 3 & 2 & 1 & 0 \\
\hline 2 & Child's actions are purposeful & 4 & 3 & 2 & 1 & 0 \\
\hline 3 & Child is aware of the surroundings & 4 & 3 & 2 & 1 & 0 \\
\hline 4 & Child is restless & 0 & 1 & 2 & 3 & 4 \\
\hline 5 & Child is inconsolable & 0 & 1 & 2 & 3 & 4 \\
\hline
\end{tabular}

\section{Secondary endpoints}

- Intraoperative hemodynamics:

Heart rate (HR)

Non-invasive BP (NIBP; systolic BP [SBP] and diastolic $\mathrm{BP}[\mathrm{DBP}]$ and mean arterial pressure [MAP])

Requirements of vasopressors, inotropes, chronotropes, and/or fluid boluses

- Incidence and duration of cerebral desaturation, defined as a decrease in regional cerebral tissue oxygenation $\left(\mathrm{rScO}_{2}\right)$ of $>20 \%$ from baseline

- Feasibility parameters:

Adequate depth of anesthesia as assessed with physiological signs (absence of movements, no rise in $\mathrm{HR}$ or $\mathrm{BP}$ of $>30 \%$ from baseline) and

bispectral index (BIS) values

Requirement of rescue medication to achieve an appropriate depth of anesthesia

Intraoperative respiratory profile (pulse

oximetry and capnography)

- arterial oxygen saturation (SaO2)

- end-tidal $\mathrm{CO}_{2}$

- Recovery parameters (measured from the stop of study treatment inhalation):

Time to open eyes, time to extubation, time to Aldrete score $\geq 9$ (readiness for discharge) [24]

Recovery index: $R I=$

$\frac{1+\text { Aldrete score at } T 5}{(2 \bullet \text { time to extubation })+\text { time to open eyes }}$ [25]

Length of Post Anesthesia Care Unit (PACU) stay

Length of hospital stay

- Other:

Levels of serum protein S100ß, IL-6, and IL-10 assessed at two time points (beginning and end of the procedure)

Radiation dose

Time of procedure

\section{Safety endpoints}

Incidence of postoperative vomiting (POV)

- On the PACU
- 12-24 h postoperatively

All other (serious) adverse events ((S)AE)

\section{Sample size}

To date, the combination of dexmedetomidine and xenon has not yet been studied in humans. The present study is designed as a pilot trial with the aim of estimating the effect size for xenon-dexmedetomidine anesthesia versus sevoflurane anesthesia with respect to the incidence of ED. In a previous study by our group in a similar patient population, we observed a $40 \%$ incidence of ED in children receiving sevoflurane. We expect an accrual of 80 children in the planned study period of 2 years. With an inclusion of a total of 80 patients, we expect to have at least 35 children in each group when anticipating possible dropouts. It is assumed that the combination of xenon-dexmedetomidine is effectively reducing the incidence of emergence delirium to $20 \%$ (i.e. a $20 \%$ absolute risk reduction). This estimated effect size is based on own data comparing sevoflurane-xenon anesthesia with sevoflurane anesthesia [11] and on a recent meta-analysis reporting an odds ratio of 0.28 (95\% confidence interval [CI] 0.190.40 ) for the effect of the intraoperative use of dexmedetomidine on the incidence of emergence delirium [26]. Based on an asymptotic 95\% CI, the expected precision (half width of the $\mathrm{CI}$ ) for the absolute risk reduction equals $21 \%$, which is given by 1.96 times the standard deviation of the absolute risk reduction.

\section{Investigational plan and treatments Preoperative treatment and monitoring}

To objectively assess emergence parameters, the children receive no benzodiazepines for anxiolysis but the parents are encouraged to accompany their children until the induction of anesthesia. After arrival in the intervention room, the following monitoring is established and recorded every $5 \mathrm{~min}$ from the pre-anesthesia period to extubation: $\mathrm{SaO}_{2}$, NIBP (SBP-DBP-MAP), $\mathrm{HR}, \mathrm{F}_{\mathrm{i}} \mathrm{O} 2$, end-tidal $\mathrm{O}_{2}$ and $\mathrm{CO}_{2}$, temperature, $\mathrm{rScO}_{2}$, and BIS.

\section{Induction of anesthesia}

Anesthesia is induced with propofol $3 \mathrm{mg} \cdot \mathrm{kg}^{-1}$, fentanyl $2 \mu \mathrm{g} \cdot \mathrm{kg}^{-1}$, and rocuronium $0.3 \mathrm{mg} \cdot \mathrm{kg}^{-1}$ as an 
intravenous bolus. Dexamethasone $0.15 \mathrm{mg} \cdot \mathrm{kg}^{-1}$ and ondansetron $0.1 \mathrm{mg} \cdot \mathrm{kg}^{-1}$ are given as standard POV prophylaxis. In the exceptional case that no intravenous line is available at induction, anesthesia is induced by mask-inhalation of sevoflurane in both study groups. In these cases, sevoflurane is immediately stopped once intravenous access has been achieved in group A (dexxenon group).

\section{Maintenance of anesthesia (anesthesia intervention)}

Eligible children are randomly assigned to one of the two study groups:

- In group A (dex-xenon group), a loading dose of 1 $\mu \mathrm{g} \cdot \mathrm{kg}^{-1}$ of dexmedetomidine is administered at induction and anesthesia is maintained by a continuous infusion of dexmedetomidine (0.5-1.0 $\mu \mathrm{g} \cdot \mathrm{kg}^{-1} \cdot \mathrm{h}$ ) and xenon $50 \%-65 \%$ in oxygen.

- In group B, anesthesia is maintained with sevoflurane alone $\left(\mathrm{FiO}_{2}=0.25-0.4\right)$.

In group $\mathrm{A}$, the infusion of dexmedetomidine and in group $B$ the sevoflurane end-tidal concentrations are titrated to achieve physiological signs suggestive of an sufficient depth of anesthesia and BIS values in the range of 40-60.

\section{Fluid management and postoperative analgesia}

Fluid management is performed according to the " $4 /$ 2/1-rule" $\left(\mathrm{mL} \cdot \mathrm{kg}^{-1} \cdot \mathrm{h}^{-1}\right)$ using a balanced crystalloid solution [27]. At the end of the procedure, all children receive paracetamol $\left(15 \mathrm{mg} \cdot \mathrm{kg}^{-1}\right)$ for postoperative analgesia.

\section{Standardized treatment of insufficient depth of anesthesia}

In case of an insufficient depth of anesthesia, the dose of the maintenance anesthetic regimen is increased (xenondex group [group A]: dexmedetomidine infusion up to $1.2 \mu \mathrm{g} \cdot \mathrm{kg}^{-1} \cdot \mathrm{hr}^{-1}$; sevo-group [group B]: increase of the age-corrected sevoflurane-concentration up to 1.5 $\mathrm{MAC})$. If still no adequate plane of anesthesia can be achieved, a bolus of propofol (1-2 $\left.\mathrm{mg} \cdot \mathrm{kg}^{-1}\right)$ or fentanyl $\left(1 \mu \mathrm{g} \cdot \mathrm{kg}^{-1}\right)$ is administered.

\section{Standardized treatment of hemodynamic instability} Isolated blood pressure drops $>20 \%$ from baseline are treated with a bolus of phenylephrine $\left(2-3 \mu \mathrm{g} \cdot \mathrm{kg}^{-1}\right)$ and/or a bolus of fluid (crystalloid $10 \mathrm{~mL} \cdot \mathrm{kg}^{-1}$ ); isolated HR declines with a bolus of atropine $\left(10-20 \mu \mathrm{g} \cdot \mathrm{kg}^{-1}\right)$ and the combination of hypotension and bradycardia with a bolus of ephedrine $\left(50-100 \mu \mathrm{g} \cdot \mathrm{kg}^{-1}\right)$.

\section{Study flow}

Visit 0: Recruitment and baseline measurement visit

After the obtainment of written parental informed consent (by one of the investigators), baseline data are evaluated and recorded (demographic data, medical and surgical history, routine clinical examination) (Figs. 1 and 2).

\section{Visit 1: General anesthesia for cardiac catheterization by investigator II}

General anesthesia will be induced and maintained as described above.

At the beginning and the end of the procedure, a blood gas sample and $2 \mathrm{~mL}$ of blood are obtained from the procedural sheet. After centrifugation, concentrations of S100 $\beta$, IL-6, and IL-100 are determined using ELISA.

\section{Visit 2: Post-anesthesia study visit PACU by investigator I}

This visit is performed from the moment of extubation until $60 \mathrm{~min}$ after extubation and consists of the following assessments:

- Assessment of HR, BP, and peripheral oxygen saturation at 5, 10, 15, 30, 45, and 60 min after extubation.

- Assessment of POV at 5, 10, 15, 30, 45, and 60 min after extubation. An emetic episode is defined as a single or continuing occurrence of vomiting or retching. Distinct episodes are defined by an interval of respite of $>1 \mathrm{~min}$. Rescue medication is offered once the patient has more than one emetic episode or on request of the patient or his parents ask.

- Aldrete score at 5, 10, 15, 30, 45, and 60 min after extubation.

- Assessment of emergence delirium by means of the "Four-point Agitation Scale" and the "PAED scale" at $5,10,15,30,45$, and 60 min after extubation.

- Assessment of the incidences of (S)AEs.

\section{Visit 3: Study end visit at the morning (12-24 h) after the procedure by investigator I}

A routine clinical examination (including $\mathrm{HR}$ and $\mathrm{BP}$ ) and the assessment of POV and (S)AE incidences are performed by investigator $I$ on the day after the procedure.

\section{Data processing and statistical methodology}

Intraoperative data are recorded manually every $5 \mathrm{~min}$ on specific case record forms (CRF). Regional cerebral tissue oxygenation $\left(\mathrm{rScO}_{2}\right)$ is electronically recorded every second. Important pre- and postoperative data are manually documented during the pre-specified visits on CRFs. All data from the CRFs are anonymized and 


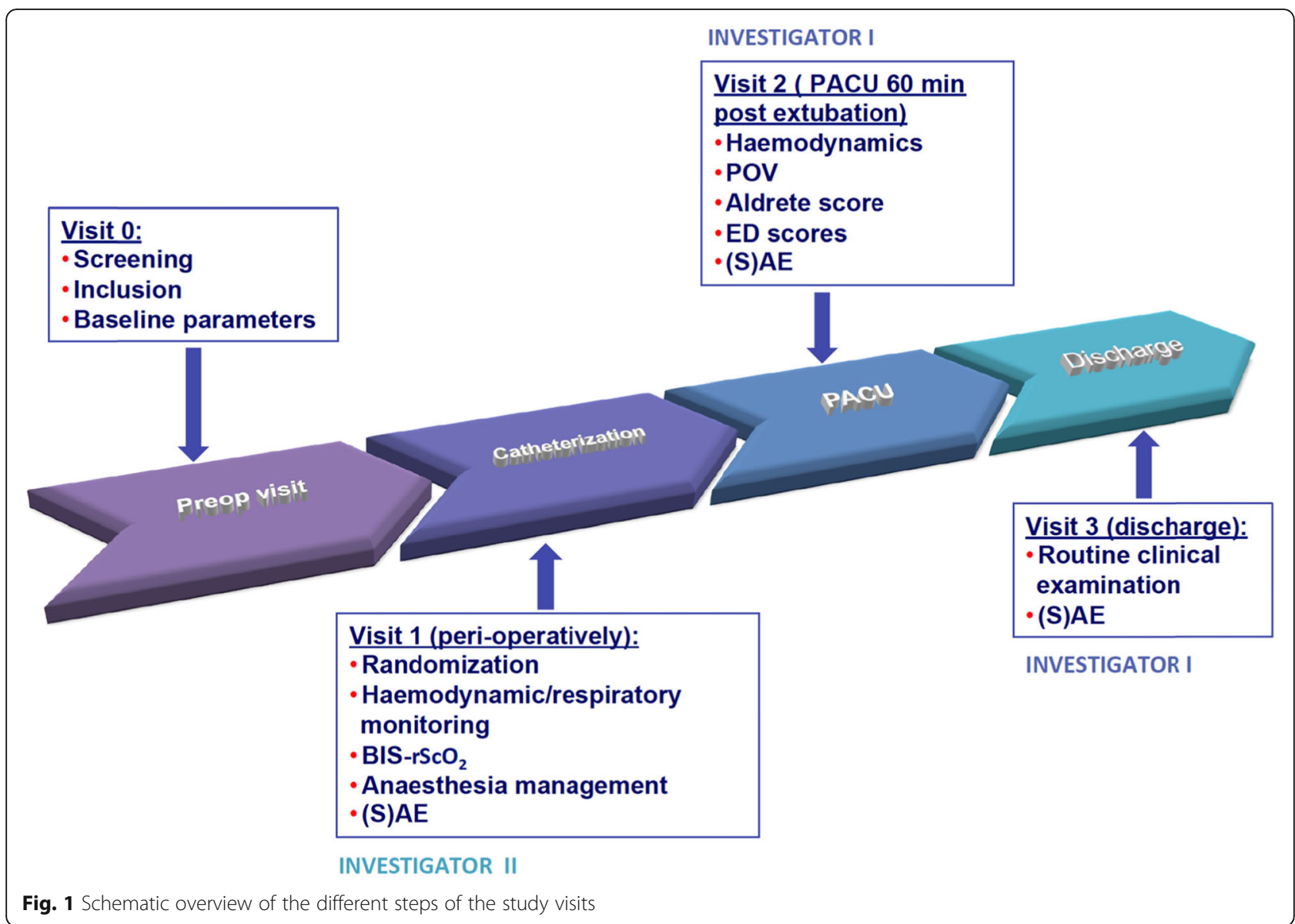

transferred into an electronic database (OpenClinica, LLC, Waltham, MA, USA). Direct access to source data and databases can be provided for trial-related monitoring, audits, EC review, and regulatory inspections.

All randomized patients will be included in the analyses. An investigator or a study nurse will review completed CRFs for completeness and correctness before digitalization and statistical analysis. Missing data will be identified, if possible drawn from source data, and filled into the CRFs. All results will be analyzed on an intention-to-treat basis. Statistical analysis will be performed using SPSS 23 software (SPSS Statistics for Windows, IBM, Armonk, NY, USA) or Prism $^{\circ}$ Software (Prism ${ }^{\bullet}$, GraphPad Software, Inc., La Jolla, CA, USA). Since the aim of the study is exploratory, summary statistics (\%, medians, means) and effect sizes with their 95\% CI will be calculated. For the primary outcome and other binary variables, the absolute risk difference and its exact 95\% CI will be reported; for normally distributed variables the difference in mean and a $t$-distribution-based 95\% CI will reported; and for non-normal or ordinal variables the Hodges-Lehmann estimate with its 95\% CI will be reported.

\section{Discussion}

ED is a frequently observed condition following pediatric anesthesia as a consequence of which a child experiences emotional suffering, restlessness, and psychomotor disturbances. It can last up to $45 \mathrm{~min}$, interferes with recovery, is associated with prolonged length of stay in the PACU and the hospital, and parental dissatisfaction. Additionally, ED can result in long-term maladaptive and behavioral disturbances (e.g. insomnia, fear, bed wetting, etc.) [2]. The diagnosis of ED remains notoriously difficult in young children. For several reasons, we opted in this study (as we did in several previous ones) for the Watcha scale as an instrument to assess the incidence of ED in this young population [11, 12, 28]. In fact, the frequently used PAED scale was derived and validated in a cohort of children with a mean age of 3.7 years [23]. In clinical practice, several items of the PAED (e.g. eye contact with the caregiver or awareness of the surrounding) appear very difficult to assess in neonates and infants. Interestingly, the latter items have recently been identified to be indicative for pain rather than for delirium [29]. Moreover, in a direct comparison of different scaling systems, the Watcha scale was found to be a more practical tool to assess ED in children after 


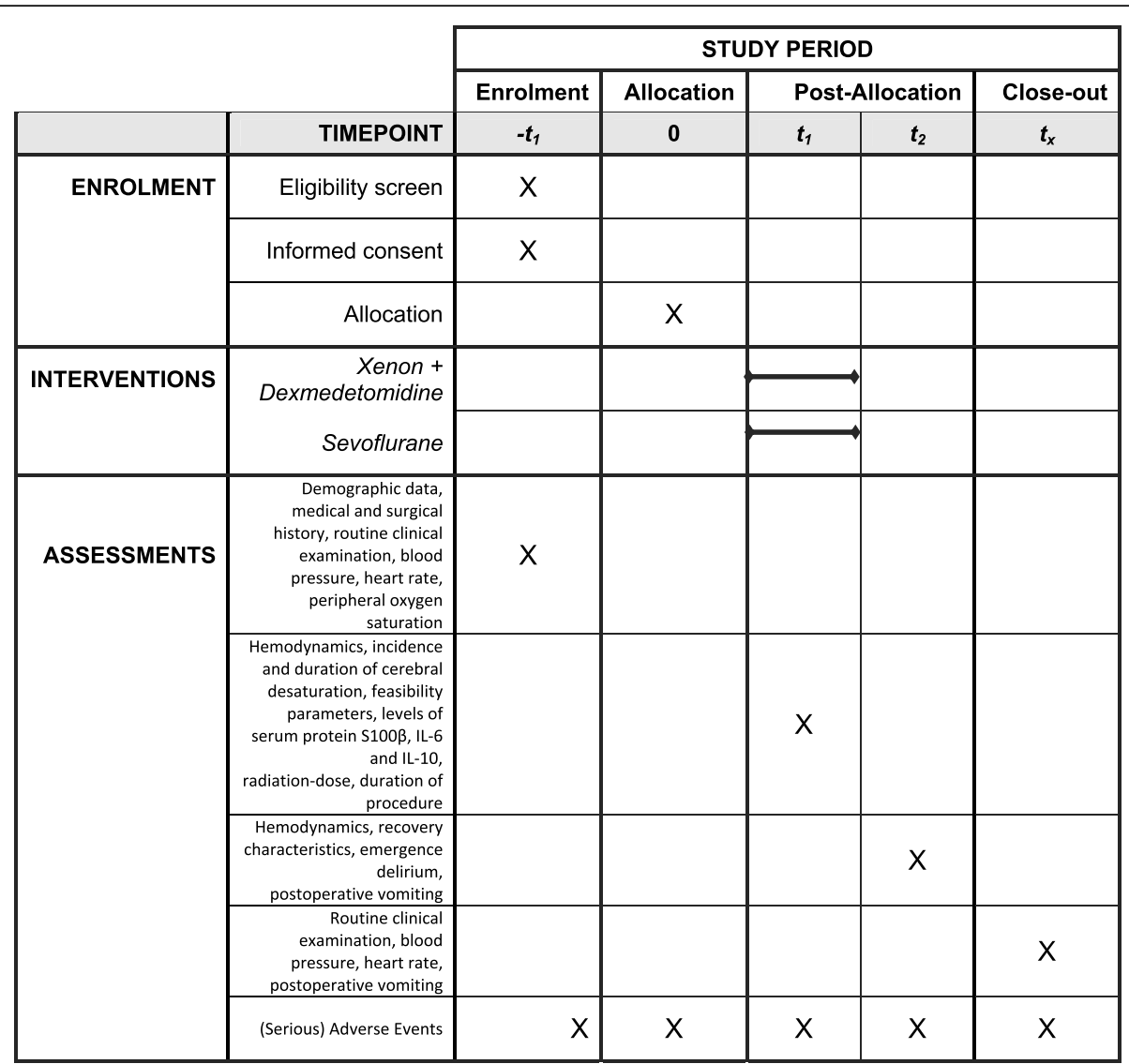

Fig. 2 Schedule of enrolment, interventions, and assessments (SPIRIT)

surgery and to have the highest overall sensitivity and specificity [22]. Note that the results of testing with the PAED scale will also be reported to allow comparison between these two instruments, an approach that is now increasingly be performed in the literature [4].

We specifically choose this patient population because: (1) the brains of these children are probably vulnerable to the adverse neuropsychiatric effects of sevoflurane; (2) younger age is a risk factor for ED; and (3) we observed a high incidence of ED in a population of children of the same age undergoing the same procedure $[4,11,30]$.

Assessing the depth of anesthesia in children remains controversial. Moreover, the validity of the BIS monitor has never been formally tested in children undergoing combined anesthesia with xenon and sevoflurane. Available evidence suggests, however, that the BIS monitor is a suitable instrument to assess the depth of xenon anesthesia. In one study, during balanced xenon anesthesia, BIS values correlated well with the clinical assessment of hypnotic depth and were within the recommended range of adequate anesthesia [31]. Moreover, Stoppe et al. demonstrated that the BIS showed a similar response to xenon as to sevoflurane-anesthesia. In the latter study, the depth of anesthesia was additionally confirmed by the measurement of auditory evoked potentials [32]. Lastly, EEG changes observed during xenon anesthesia resembled closely those instigated by propofol [33]. Furthermore, although age-related changes in BIS values in children were observed, it has been suggested that the BIS is a reliable monitor of depth of anesthesia at least in children aged $>1$ year, showing better performance with increasing age [34, 35]. Note that in our study, the adequacy of depth of anesthesia will primarily be assessed by physiological signs (absence of HR or BP increases and involuntarily movements) and the BIS monitor is only an additional tool to quantify depth of anesthesia.

We will assess the peri-interventional release of S100 $\beta$ as a secondary endpoint. The astroglial receptor protein $\mathrm{S} 100 \beta$ is involved in several cellular processes such as neuronal differentiation, axonal growth, and calcium homeostasis [36]. Increased levels of $S 100 \beta$ in the peripheral blood have been suggested to indicate an increased permeability of the blood-brain barrier and found to be associated with neurotrauma, cerebral ischemia, cardiac arrest, and cardiac surgery [37]. In neonates suffering from $\mathrm{CHD}$, levels of $\mathrm{S} 100 \beta$ were described to be inversely related to cerebral blood flow and to 
mortality [38]. In children undergoing general anesthesia for cardiac catheterization, we recently reported early neurocognitive deficits that were paralleled by an increase in S100 $\beta$ blood levels [12]. In adult patients undergoing cardiac surgery, high levels of S100 $\beta$ were observed to be correlated with adverse neuropsychological and psychiatric outcomes [39] and to predict the occurrence of postoperative delirium [36]. The exact mechanisms resulting in a perioperative/peri-interventional increase in $S 100 \beta$ remain still unknown but might involve neuro-inflammatory pathways with astrocytes being stimulated by cytokines originating from systemic inflammation to release S100 $\beta$. Therefore, we will also assess the peri-interventional release of the pro- and anti-inflammatory cytokines IL-6 and IL-10 in our population. We acknowledge that the findings will be exploratory, solely describe associations, and should not be used to assess causation.

The current standard of care for general anesthesia in childhood overall, and for cardiac catheterization more specifically, is sevoflurane. The use of sevoflurane has been linked to an increased incidence of ED [4]. Moreover, sevoflurane was listed by the FDA as potentially neurotoxic when used in children aged $<3$ years (during the episode of rapid brain development). In contrast, dexmedetomidine and xenon have no proven neurotoxicity in preclinical research and even have the potential to reduce the incidence of ED [40]. The combination of both drugs has never been described as a balancedanesthesia technique in children.

The exposure of young children to the combination of dexmedetomidine and xenon will provide the first human data of a pediatric anesthesia regimen without the use of any drugs that were indicated by the FDA to potentially impair neurodevelopmental outcome when repeatedly or lengthily used for general anesthesia and sedation during surgeries or procedures in children aged $<3$ years or in pregnant women during their third trimester of pregnancy.

We acknowledge that our study is subject to several limitations. First, the attending anesthesiologist cannot be blinded for the interventional treatment. However, the primary outcome will be assessed by an investigator unaware of the treatment allocation ("observer blinded"). Second, it is not defensible to extrapolate the feasibility of xenon and dexmedetomidine as adjuncts for general anesthesia in young children undergoing cardiac catheterization to surgical settings with severe painful stimulation. Third, this randomized controlled trial is not designed to prove the potential superiority of xenon/ dexmedetomidine versus sevoflurane anesthesia with regard to the incidence of ED. To perform a trial with this purpose, a precise idea of the expected ED incidences is necessary for appropriate sample size estimation. The current study will deliver data allowing to design an adequately powered randomized controlled trial addressing this research question. Note that the current study is also not sufficiently powered for all secondary endpoints, which should, therefore, be considered as purely explorative and hypothesis-generating.

In conclusion, this pilot trial will be performed to investigate the incidence of ED in an anesthesia regimen that does not involve sevoflurane. Moreover, we will study the feasibility and the safety of combined dexmedetomidine-xenon anesthesia in children.

\section{Benefits for the participating patients}

There is no guarantee that the combination of dexmedetomidine and xenon, instead of standard treatment with mono-sevoflurane, will result in less ED or any other medical advantage to the participant.

\section{Safety issues}

Standard hemodynamic monitoring in the setting of a fully equipped cardiac catheterization room enables immediate detection and treatment of AEs. Xenon inhalation or dexmedetomidine-infusion will be stopped immediately in case of a life-threatening deterioration of a study patient. All study participants will be closely monitored by the study team for the occurrence of (S)AEs. Moreover, the inclusion of each individual patient into the study is indicated in the electronic hospital information system and hence visible to all physicians and nurses involved in the care of this patient. This facilitates reporting of (S)AEs to the principal investigator. The principal investigator will report suspected unexpected serious adverse reactions (SUSARs) to the federal health authorities. Furthermore, study data will be regularly checked for safety by an independent clinical pediatric cardiologist who is not involved in this clinical trial. Lastly, the study is regularly audited by the clinical trials center of the sponsor (UZ Leuven).

\section{Data handling}

All information and data relating to the study will be treated as confidential and such information will not be disclosed to any third parties or used for any purpose other than the performance of the study. The collection, processing, and disclosure of personal data such as patient health and medical information are in compliance with applicable European and national laws on personal data protection and the processing of personal data. Our data will be coded (continued link between the data and the individual who provided it). The participant's name or other identifiers will be stored separately (in the investigators' site file) and replaced with a unique code to create a new identity for the subject. Data will be entered into an electronic case record form (OpenClinica, 
Waltham, MA, USA). The study will be monitored by the clinical trials center of the UZ Leuven.

\section{Trial status}

a. Protocol version number and date: protocol SDDXP 2 06-08-2018, approved by the ethics committee on 18 August 2018

b. Date recruitment began: 18 December 2018

c. Current status: 40 out of 80 patients have been recruited at the time of writing the manuscript

d. Approximate date when recruitment will complete: 30 June 2020

\section{Supplementary information}

Supplementary information accompanies this paper at https://doi.org/10. 1186/s13063-020-4231-5.

Additional file 1. SPIRIT 2013 Checklist: Recommended items to address in a clinical trial protocol.

Additional file 2. World Health Organization trial registration dataset. Additional file 3. Informed consent.

\section{Abbreviations}

BIS: Bispectral Index; BL: Baseline; CHD: Congenital heart disease; CRF: Case report form; DBP: Diastolic blood pressure; ED: Emergence delirium; FDA: United States Food and Drug Administration; $\mathrm{F}_{0} \mathrm{O}_{2}$ : Fraction inspired oxygen; HR: Heart rate; IL: Interleukin; KU: Katholieke Universiteit; MAC: Minimal alveolar concentration; NIBP: Non-invasive blood pressure; OR: Odds ratio; PACU: Post Anesthesia Care Unit; PAED: Pediatric Anesthesia Emergence Delirium Scale; POV: Postoperative vomiting; RI: Recovery Index $\mathrm{rSCO}_{2}$ : Regional cerebral tissue oxygenation; SBP: Systolic blood pressure; $\mathrm{S}_{2} \mathrm{O}_{2}$ : Arterial oxygen saturation; (S)AE: (Serious) adverse event;

SUSAR: Suspected unexpected serious adverse reaction

\section{Acknowledgements}

We would like to thank our study coordinator Christel Huygens for her invaluable assistance in performing this clinical trial.

\section{Authors' contributions}

SD and SR conceptualized and designed this study, provide leadership for study conduct, acquire study-related data and wrote this manuscript; LD, $\mathrm{FDB}, \mathrm{BC}, \mathrm{MG}$, and MVDV contributed to the design of the study and the conduct of the intervention; SD, SR, and SF contributed to the design and development of the analysis; SF contributed substantially to writing this manuscript. All authors read and approved the final manuscript.

\section{Availability of data and materials}

Intraoperative data are recorded manually every 5 min on specific case record forms (CRF). Regional cerebral tissue oxygenation $\left(\mathrm{rSCO}_{2}\right)$ is electronically recorded every second. Important pre- and postoperative data are manually documented during the pre-specified visits on CRFs. All data from the CRFs are transferred into an electronic database (OpenClinica, LLC, Waltham, MA, USA). Direct access to source data and databases can be provided for trial-related monitoring, audits, EC review, and regulatory inspections. The datasets used and/or analyzed during the present study are available from the corresponding author on reasonable request.

\section{Ethics approval and consent to participate}

The protocol (version SD-DXP: 2 06-08-2018) and related documents were approved by the Ethics Committee of the University Hospitals Leuven (S61690, 18 August 2018). Informed consent will be obtained from all study participants.

\section{Consent for publication}

Not applicable.

\section{Competing interests}

The study is being funded solely by institutional and departmental sources. In the past, SR received an unrestricted research grant from Air Liquide. The sponsor of this clinical trial is UZ Leuven, Belgium. Neither sponsor or funders have any role in study design, collection/management/analysis/ interpretation of data, writing the report, decision to submit the report for publication, and have no authority over any of these activities.

\section{Author details}

'Department of Anesthesiology, University Hospitals Leuven, Herestraat 49, 3000 Leuven, Belgium. ²Department of Pediatric and Congenital Cardiology, University Hospitals Leuven, Leuven, Belgium. ${ }^{3}$ I-Biostat, KU Leuven University of Leuven, Leuven, Belgium. ${ }^{4}$ Department of Cardiovascular Sciences, Katholieke Universiteit (KU) Leuven, Leuven, Belgium.

Received: 7 November 2019 Accepted: 4 March 2020

Published online: 03 April 2020

References

1. Wong DDL, Bailey CR. Emergence delirium in children. Anaesthesia. 2015; 70(4):383-7.

2. Dahmani S, Delivet $H$, Hilly J. Emergence delirium in children: an update. Curr Opin Anaesthesiol. 2014:27(3):309-15.

3. Chandler JR, Myers D, Mehta D, Whyte E, Groberman MK, Montgomery CJ, et al. Emergence delirium in children: a randomized trial to compare total intravenous anesthesia with propofol and remifentanil to inhalational sevoflurane anesthesia. Paediatr Anaesth. 2013;23(4):309-15.

4. Costi D, Cyna AM, Ahmed S, Stephens K, Strickland P, Ellwood J, et al. Effects of sevoflurane versus other general anaesthesia on emergence agitation in children. Costi D, editor. Cochrane Database Syst Rev. 2014;9: CD007084.

5. Law LS-C, Lo EA-G, Gan TJ. Xenon anesthesia: a systematic review and meta-analysis of randomized controlled trials. Anesth Analg. 2016;122(3): 678-97.

6. Van Hese L, Al tmimi L, Devroe S, Sanders RD, Fieuws S, Rex S. Neuroprotective properties of xenon in different types of CNS injury. Br J Anaesth. 2018;121(6):1365-68.

7. Ma D, Williamson $P$, Januszewski $A$, Nogaro $M-C$, Hossain $M$, Ong $L P$, et al. Xenon mitigates isoflurane-induced neuronal apoptosis in the developing rodent brain. Anesthesiology. 2007;106(4):746-53.

8. Nakata Y, Goto T, Ishiguro Y, Terui K, Kawakami H, Santo M, et al. Minimum alveolar concentration (MAC) of xenon with sevoflurane in humans. Anesthesiology. 2001;94(4):611-4.

9. Mapleson WW. Effect of age on MAC in humans: a meta-analysis. $\mathrm{Br} J$ Anaesth. 1996:76(2):179-85.

10. Dingley J, Tooley J, Porter H, Thoresen M. Xenon provides short-term neuroprotection in neonatal rats when administered after hypoxia-ischemia. Stroke. 2006;37(2):501-6.

11. Devroe $S$, Meeusen $R$, Gewillig M, Cools B, Poesen $K$, Sanders R, et al. Xenon as an adjuvant to sevoflurane anesthesia in children younger than 4 years of age, undergoing interventional or diagnostic cardiac catheterization: A randomized controlled clinical trial. Paediatr Anaesth. 2017:27(12):1210-9.

12. Devroe S, Lemiere J, Van Hese L, Gewillig M, Boshoff D, Poesen K, et al. The effect of xenon-augmented sevoflurane anesthesia on intraoperative hemodynamics and early postoperative neurocognitive function in children undergoing cardiac catheterization: A randomized controlled pilot trial. Paediatr Anaesth. 2018;28(8):726-38.

13. Pan W, Wang $Y$, Lin L, Zhou G, Hua X, Mo L. Outcomes of dexmedetomidine treatment in pediatric patients undergoing congenital heart disease surgery: a meta-analysis. Paediatr Anaesth. 2016;26(3):239-48.

14. Schwartz LI, Twite M, Gulack B, Hill K, Kim S, Vener DF. The perioperative use of dexmedetomidine in pediatric patients with congenital heart disease: an analysis from the Congenital Cardiac Anesthesia Society-Society of Thoracic Surgeons Congenital Heart Disease Database. Anesth Analg. 2016;123(3): 715-21.

15. Ahmed SS, Unland TL, Slaven JE, Nitu ME. Dexmedetomidine versus propofol: is one better than the other for MRI sedation in children? J Pediat Intensive Care. 2017;6(2):117-22. 
16. Fragen RJ, Fitzgerald PC. Effect of dexmedetomidine on the minimum alveolar concentration (MAC) of sevoflurane in adults age 55 to 70 years. J Clin Anesth. 1999;11(6):466-70.

17. He L, Wang $X$, Zheng S. Effects of dexmedetomidine on sevoflurane requirement for $50 \%$ excellent tracheal intubation in children: a randomized, double-blind comparison. Paediatr Anaesth. 2014;24(9):987-93.

18. Sanders RD, SUN P, PATEL S, Li M, Maze M, Ma D. Dexmedetomidine provides cortical neuroprotection: impact on anaesthetic-induced neuroapoptosis in the rat developing brain. Acta Anaesthesiol Scand. 2010; 54(6):710-6.

19. FDA Drug Safety Communication: FDA review results in new warnings about using general anesthetics and sedation drugs in young children and pregnant women. 2016. http://www.fda.gov/downloads/Drugs/DrugSafety/ UCM533197.pdf. Accessed 5 Mar 2017.

20. Chan A-W, Tetzlaff JM, Altman DG, Laupacis A, Gøtzsche PC, Krleža-Jerić K, et al. SPIRIT 2013 statement: defining standard protocol items for clinical trials. Washington, DC: American College of Physicians; 2013. p. 200-7.

21. Watcha MF, Ramirez-Ruiz M, White PF, Jones MB, Lagueruela RG, Terkonda RP. Perioperative effects of oral ketorolac and acetaminophen in children undergoing bilateral myringotomy. Can J Anaesth. 1992;39(7):649-54.

22. Bajwa SA, Costi D, Cyna AM. A comparison of emergence delirium scales following general anesthesia in children. Pediatr Anesth. 2010;20(8):704-11.

23. Sikich N, Lerman J. Development and psychometric evaluation of the pediatric anesthesia emergence delirium scale. Anesthesiology. 2004;100(5): $1138-45$.

24. Aldrete JA. The post-anesthesia recovery score revisited. J Clin Anesth. 1995; 7(1):89-91.

25. Rossaint R, Reyle-Hahn M, Schulte Am Esch J, Scholz J, Scherpereel P, Vallet $B$, et al. Multicenter randomized comparison of the efficacy and safety of xenon and isoflurane in patients undergoing elective surgery. Anesthesiology. 2003;98(1):6-13.

26. Pickard A, Davies P, Birnie K, Beringer R. Systematic review and metaanalysis of the effect of intraoperative $a_{2}$-adrenergic agonists on postoperative behaviour in children. Br J Anaesth. 2014;112(6):982-90.

27. Oh TH. Formulas for calculating fluid maintenance requirements. Anesthesiology. 1980;53(4):351.

28. Devroe S, Lemiere J, van de Velde M, Gewillig M, Boshoff D, Rex S. Safety and feasibility of xenon as an adjuvant to sevoflurane anaesthesia in children undergoing interventional or diagnostic cardiac catheterization: study protocol for a randomised controlled trial. Trials BioMed Central. 2015;16(1):74.

29. Somaini M, Engelhardt T, Fumagalli R, Ingelmo PM. Emergence delirium or pain after anaesthesia-how to distinguish between the two in young children: a retrospective analysis of observational studies. Br J Anaesth. 2016;116(3):377-83.

30. Mason KP. Paediatric emergence delirium: a comprehensive review and interpretation of the literature. Br J Anaesth. 2017;118(3):335-43.

31. Fahlenkamp AV, Krebber F, Rex $S$, Grottke O, Fries M, Rossaint R, et al. Bispectral index monitoring during balanced xenon or sevoflurane anaesthesia in elderly patients. Eur J Anaesthesiol. 2010;27(10):906-11.

32. Stoppe C, Peters D, Fahlenkamp AV, Cremer J, Rex S, Schälte G, et al. aepEX monitor for the measurement of hypnotic depth in patients undergoing balanced xenon anaesthesia. Br J Anaesth. 2012;108(1):80-8.

33. Laitio RM, Kaskinoro K, Särkelä MOK, Kaisti KK, Salmi E, Maksimow A, et al. Bispectral index, entropy, and quantitative electroencephalogram during single-agent xenon anesthesia. Anesthesiology. 2008;108(1):63-70.

34. Davidson AJ, Kwok T. Performance of BIS in children using the paediatric BIS Quattro sensor. Anaesth Intensive Care. 2008;36(6):807-13.

35. Sciusco A, Standing JF, Sheng Y, Raimondo P, Cinnella G, Dambrosio M. Effect of age on the performance of bispectral and entropy indices during sevoflurane pediatric anesthesia: a pharmacometric study. Paediatr Anaesth. 2017:27(4):399-408.

36. Tmimi Al L, van de Velde M, Meyns B, Meuris B, Sergeant $P$, Milisen $K$, et al. Serum protein S100 as marker of postoperative delirium after off-pump coronary artery bypass surgery: secondary analysis of two prospective randomized controlled trials. Clin Chem Lab Med. 2016;54(10):1671-80.

37. Abu-Sultaneh S, Hehir DA, Murkowski K, Ghanayem NS, Liedel J, Hoffmann $\mathrm{RG}$, et al. Changes in cerebral oxygen saturation correlate with S100B in infants undergoing cardiac surgery with cardiopulmonary bypass. Pediatr Crit Care Med. 2014;15(3):219-28.

38. Bokesch PM, Appachi E, Cavaglia M, Mossad E, Mee RBB. A glial-derived protein, $\mathrm{S} 100 \mathrm{~B}$, in neonates and infants with congenital heart disease: evidence for preexisting neurologic injury. Anesth Analg. 2002;95(4):889-92.
39. Herrmann M, Vos P, Wunderlich MT, de Bruijn CH, Lamers KJ. Release of glia tissue-specific proteins after acute stroke: a comparative analysis of serum concentrations of protein S-100B and glial fibrillary acidic protein. Stroke. 2000;31(11):2670-7.

40. Alam A, Suen KC, Hana Z, Sanders RD, Mervyn M, Ma D. Neuroprotection and neurotoxicity in the developing brain: an update on the effects of dexmedetomidine and xenon. Neurotoxicol Teratol. 2017;60:102-16.

\section{Publisher's Note}

Springer Nature remains neutral with regard to jurisdictional claims in published maps and institutional affiliations.
Ready to submit your research? Choose BMC and benefit from:

- fast, convenient online submission

- thorough peer review by experienced researchers in your field

- rapid publication on acceptance

- support for research data, including large and complex data types

- gold Open Access which fosters wider collaboration and increased citations

- maximum visibility for your research: over $100 \mathrm{M}$ website views per year

At BMC, research is always in progress.

Learn more biomedcentral.com/submissions 